\title{
Highly efficient and stable coupling of kilowatt-level continuous wave laser into hollow-core fibers
}

\author{
Yulong Cui (崔宇龙) $)^{1,2}$, Wei Huang (黄 威) ${ }^{1,2}$, Zhiyue Zhou (周智越) $)^{1,2}$, Hao Li (李 昊) $)^{1,2}$, Meng Wang (王 蒙) $)^{1,2,3}$ ， \\ Zilun Chen (陈子伦) $)^{1,2,3}$, and Zefeng Wang (王泽锋) $)^{1,23^{*}}$ \\ ${ }^{1}$ College of Advanced Interdisciplinary Studies, National University of Defense Technology, Changsha 410073, China \\ ${ }^{2}$ State Key Laboratory of Pulsed Power Laser Technology, Changsha 410073, China \\ 3 Hunan Provincial Key Laboratory of High Energy Laser Technology, Changsha 410073, China
}

*Corresponding author: zefengwang_nudt@163.com

Received December 2, 2021 | Accepted January 18, 2022 | Posted Online February 14, 2022

\begin{abstract}
Fiber gas lasers based on gas-filled hollow-core fibers (HCFs) perfectly combine the advantages of fiber lasers and gas lasers and have obtained fast development in the past years. However, stable and efficient coupling of high-power pump lasers into the HCFs is one of the key problems to be solved. In this paper, we study the coupling of high-power continuous wave fiber lasers into anti-resonant HCFs through an end-cap. By optimizing the splicing parameters, a maximum laser power of $1167 \mathrm{~W}$ was injected into the 1-m-long HCFs, and $1021 \mathrm{~W}$ was obtained at the output end, giving a total transmission efficiency of $\sim 87.5 \%$. A more than $1 \mathrm{~h}$ test showed the stability of such a coupling method. Meanwhile, the laser beam quality was well maintained. This work opens new opportunities for stable and highly efficient coupling of high-power lasers into HCFs, which is significant for its applications in many other fields besides high-power fiber gas lasers, such as high-power laser delivering.
\end{abstract}

Keywords: hollow-core fibers; end-caps; high power; coupling methods. DOI: 10.3788/COL202220.040602

\section{Introduction}

Different from the solid-state fiber using total reflection, the hollow-core fiber (HCF) can confine light into the hollow core with 10 to $100 \mu \mathrm{m}$ diameters due to its special cladding structure, and it can also be filled with various kinds of gases, which provides an ideal environment for the interaction between the laser and gases due to its hollow-core structure ${ }^{[1]}$. Thus, a novel kind of laser named a fiber gas laser (FGL) was developed ${ }^{[2]}$, and great progresses have been made in this kind of laser source ${ }^{[3-14]}$. However, the power level can hardly breach $10 \mathrm{~W}$; one main cause is the lack of stable and high-efficiency coupling methods of high-power lasers into HCFs. Besides, HCFs can confine almost all of the energy into the hollow core, similar to freespace transmission, which improves the damage threshold and weakens the nonlinear effect. Therefore, HCFs have great potential for high-power transmission. Some efficient highpower transmission experiments have been reported such as $300 \mathrm{~W}$ without cooling in an anti-resonant $\mathrm{HCF}^{[15]}$ and $900 \mathrm{~W}$ with water cooling in a Kagome photonic crystal fiber $(\mathrm{PCF})^{[16]}$, but they were not stable enough for long-term working. A highefficiency and stable coupling method for high-power coupling into HCFs is needed.
There have been many researches about the power coupling into the HCF. One possible method is the splicing between the solid-core fiber and HCF. In 2005, one all-fiber gas cavity based on a hollow-core PCF (HC-PCF) with the splicing loss of 1 to $2 \mathrm{~dB}$ was reported ${ }^{[17]}$. The splicing loss of other reports about the splicing between the solid-core fiber and HC-PCF was about $1.8 \mathrm{~dB}^{[18]}$ and $0.79 \mathrm{~dB}^{[19]}$. In 2014, the intermediate fiber was used between the HC-PCF and single mode fiber (SMF), and the overall insertion loss was $0.73 \mathrm{~dB}^{[20]}$. The main reason for the insertion loss is the mismatch of the mode field. Based on the splicing methods between the SMF and HC-PCF, several all-fiber Raman FGLs were reported ${ }^{[21-25]}$. However, the output power was only the level of several watts, and, due to the high insertion loss, heat accumulation easily occurred at the splicing point. For the HCFs with larger core diameters like Kagome HCFs and anti-resonant HCFs, it is more difficult to realize the match of the mode field. In 2016, the insertion loss of $0.48 \mathrm{~dB}$ was reported by splicing the tapered Kagome PCF and $\mathrm{SMF}^{[26]}$. Another tapering method named nanospike was reported $^{[27]}$. By tapering the SMF into several hundreds of nanometers, the mode field increased, and the coupling efficiency was as high as $87.8 \%$. Referring to this method, our group reported several quasi-all-fiber gas lasers by inserting a tapered SMF into 
an anti-resonant $\mathrm{HCF}^{[28-30]}$. However, the diameter after tapering was still a few microns, which still causes the mismatch of the mode field, and the coupling efficiency was unsatisfactory. This method is also not suitable for higher power due to its small core size. Recently, a new two-step reverse tapering approach was reported. By reversely tapering the SMFs first and subsequently thermally expanding their core, mode field matching was realized, and the loss was $0.23 \mathrm{~dB}$ between the SMF and $\mathrm{PCF}^{[31]}$ and $0.88 \mathrm{~dB}$ for the two joints for SMF/HCF/SMF ${ }^{[32]}$. All of above methods were not carried out in a high-power transmission system. Our group proposed a high-efficiency coupling method using HCF end-caps, which has stable coupling of high-power capacity, and it has been tested in a $260 \mathrm{~W}$ power transmission system without cooling ${ }^{[33]}$.

In this study, a kilowatt $(\mathrm{kW})$-level coupling experiment was carried out by using HCF end-caps. By optimizing the splicing parameters and using the water cooling system, $1021 \mathrm{~W}$ transmission power was obtained using 1-m-long anti-resonant HCFs under $1167 \mathrm{~W}$ injected power into the HCF with a total efficiency as high as $87.5 \%$. Through $1 \mathrm{~h}$ testing for the power stability and beam quality, this method can be proved to be an efficient and stable coupling method at $915 \mathrm{~W}$. This experiment lays the foundation for high-power FGLs and high-power transmission in the HCF.

\section{Experiment Setup}

The schematic of the power transmission system is shown in Fig. 1(a). The fiber laser source emits a CW laser with the maximum output power of $1500 \mathrm{~W}$ at the wavelength of $1070 \mathrm{~nm}$. The output fiber is a $20 \mu \mathrm{m}$ core solid fiber fabricated with an end-cap, and the beam quality $M^{2}$ of the laser source is 1.17 at the maximum output power of $1500 \mathrm{~W}$. The power is coupled into the HCF using a coupling system composed by two planoconvex lenses and two reflectors, and all of them are fixed on the three-axis adjustment platform. The lenses and the reflectors are all custom made for high-power transmission using the material of Corning $7980 \mathrm{OD}$ and ultraviolet-fused quartz and coated at the corresponding wavelength band. The focus of the two lenses
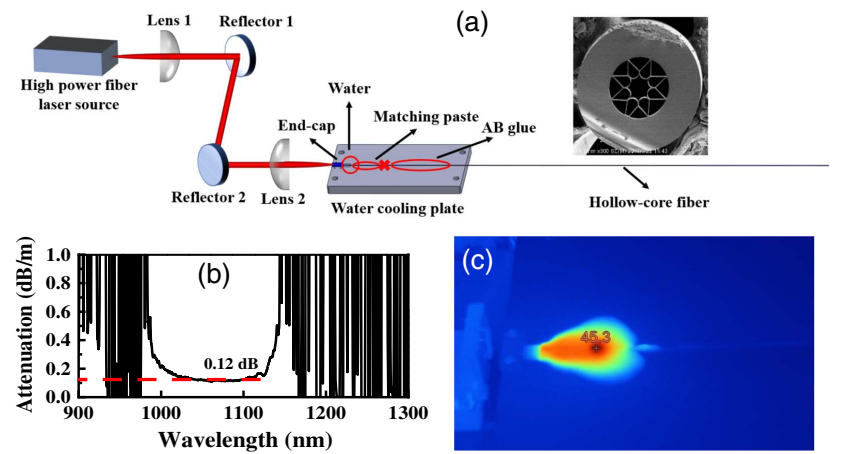

Fig. 1. (a) Experiment setup of the power transmission system. Inset: crosssection electronic micrograph of HCF. (b) Transmission loss of HCF used at $1 \mu \mathrm{m}$ wavelength band; (c) thermal image of the input end.
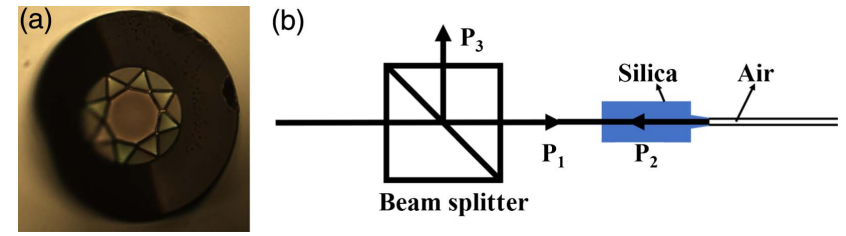

Fig. 2. (a) Cross-section micrograph of HCF after splicing with end-cap; (b) schematic of test for Fresnel reflection.

is $30 \mathrm{~mm}$ with the transmission higher than $99.9 \%$, and the reflection of the reflector is higher than $99.8 \%$. The HCF is supported by Bath University. The cross section of the HCF is shown in the inset picture of Fig. 1(a), where the core diameter is around $46 \mu \mathrm{m}$, with eight ice-cream capillaries surrounding the core, which form the anti-resonant region. The thickness of the capillaries is around $1.3 \mu \mathrm{m}$, and the outer diameter of the cladding is $280 \mu \mathrm{m}$. Fiber loss at $1070 \mathrm{~nm}$ is around $0.12 \mathrm{~dB} / \mathrm{m}$, as shown in Fig. 1(b).

The input end of the HCF is fabricated with an end-cap for fixing and protection of the HCF. The end-cap is custom made by a company from China. The HCF with the end-cap is fixed on the water cooling plate designed by us. An approximately $25-$ $\mathrm{cm}$-long HCF is water cooled, which contains $10 \mathrm{~cm}$ without coating and $15 \mathrm{~cm}$ with coating, and the red cross sign in Fig. 1(a) is the junction between the cladding and the coating. The cladding part is applied with matching paste to filter the cladding light, a small distance of HCF splicing with an endcap is suspended, and water is used to realize cooling. The front of the coating part is applied with $\mathrm{AB}$ glue for a better cooling effect. At the transmission power of $1 \mathrm{~kW}$, apart from the cladding part applied with matching paste, as shown in Fig. 1(c), the temperature is $45.3^{\circ} \mathrm{C}$, and the whole $1-\mathrm{m}$-long HCF has no obvious temperature rise.

The HCF end-cap is fabricated with a large diameter splicing system. Due to the special hollow-core structure of the HCF, the shape of the core changes when heated, as shown in Fig. 2(a), where the negative curvature of the core is destroyed, and the ice-cream capillaries are changed to triangles. Either the core diameter or the mode field diameter becomes larger. To avoid excessive deformation, the heating temperature is controlled strictly. The core of the HCF is filled with air, and, after splicing with HCF, there is a surface between the silica and the air, which causes Fresnel reflection. The testing system in Fig. 2(b) is used to measure the Fresnel reflection. $P_{1}$ is the power that is injected into the end-cap, and $P_{2}$ is the back power. A non-polarizing beam splitter is used, and the measured (reflection/transmission) split ratio is 5:95. Through the measurement of $P_{3}, P_{2}$ can be estimated, and the Fresnel reflection we measured is around $3.5 \%$.

\section{Results}

The power transmission results for the $\mathrm{kW}$-level power coupling experiment are shown in Fig. 3. The left axis shows the 


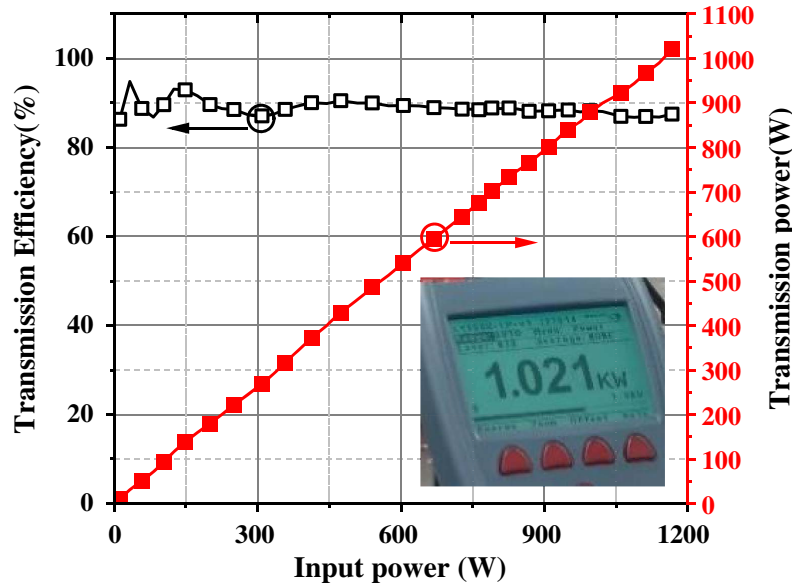

Fig. 3. Measured transmission power and transmission efficiency versus input power.

transmission efficiency, which is the transmission power after the HCF divides the power of the laser source, and the right axis shows the transmission power. The highest transmission power is $1021 \mathrm{~W}$, as the laser source power is $1167 \mathrm{~W}$, and the total transmission efficiency is as high as $87.5 \%$. Coupling efficiency has a fluctuation under the input power of $300 \mathrm{~W}$, which is because the power meter has a big fluctuation at a relatively low power level, and the efficiency is uncertain. When the input power is above $300 \mathrm{~W}$, the transmission efficiency is stable at around $87.5 \%$ when the power of the laser source is increased. Considering the loss of the lenses and the reflectors of around $0.03 \mathrm{~dB}$, the Fresnel reflection of the HCF end-cap of around $0.15 \mathrm{~dB}$, and the fiber loss of around $0.12 \mathrm{~dB}$, the estimated coupling efficiency is about $94 \%$. The coupling efficiency of $94 \%$ indicates that this is an efficient coupling method. Because the ratio of focal lengths of two lenses we used is one, the mode field diameter of the beam injected into the hollow core should be the same as the mode field of the output fiber of the laser source. But, in practice, power monitoring at the output end is used, the focal lengths are changed to ensure the highest coupling efficiency, and the real mode field diameter of the beam after lenses is very close to that of the HCF, so the coupling efficiency is as high as $94 \%$. The other $6 \%$ loss may be the mismatch of the mode field shape, and some light transmits in the cladding region with higher loss.

The power stability is measured, and the results are shown in Fig. 4. The power of the laser source has a fluctuation up and down because of the periodic working of the water cooling, and the change of the fiber temperature will influence the output power. For the purpose of protecting the system, we do not measure the power stability at the maximum transmission power. As the transmission power is $950 \mathrm{~W}$, during the testing time of $60 \mathrm{~min}$, the transmission power is declined from $950 \mathrm{~W}$ to $930 \mathrm{~W}$, which is due to heat accumulation; as the temperature of the HCF changed, there was a micro deformation for the $\mathrm{HCF}$, which caused the decline of the efficiency. It is possible because, as the heat accumulated, the front end of the HCF

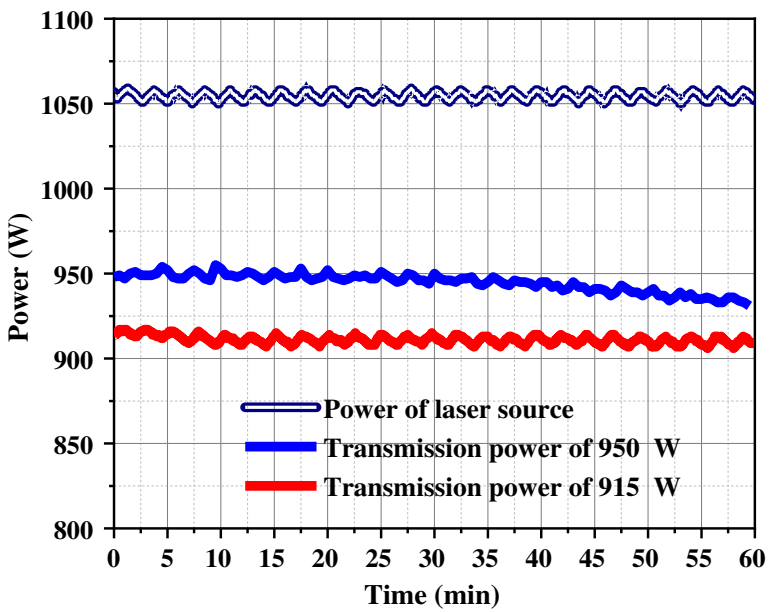

Fig. 4. Power stability of the laser source and the transmission power of $915 \mathrm{~W}$ and $950 \mathrm{~W}$.
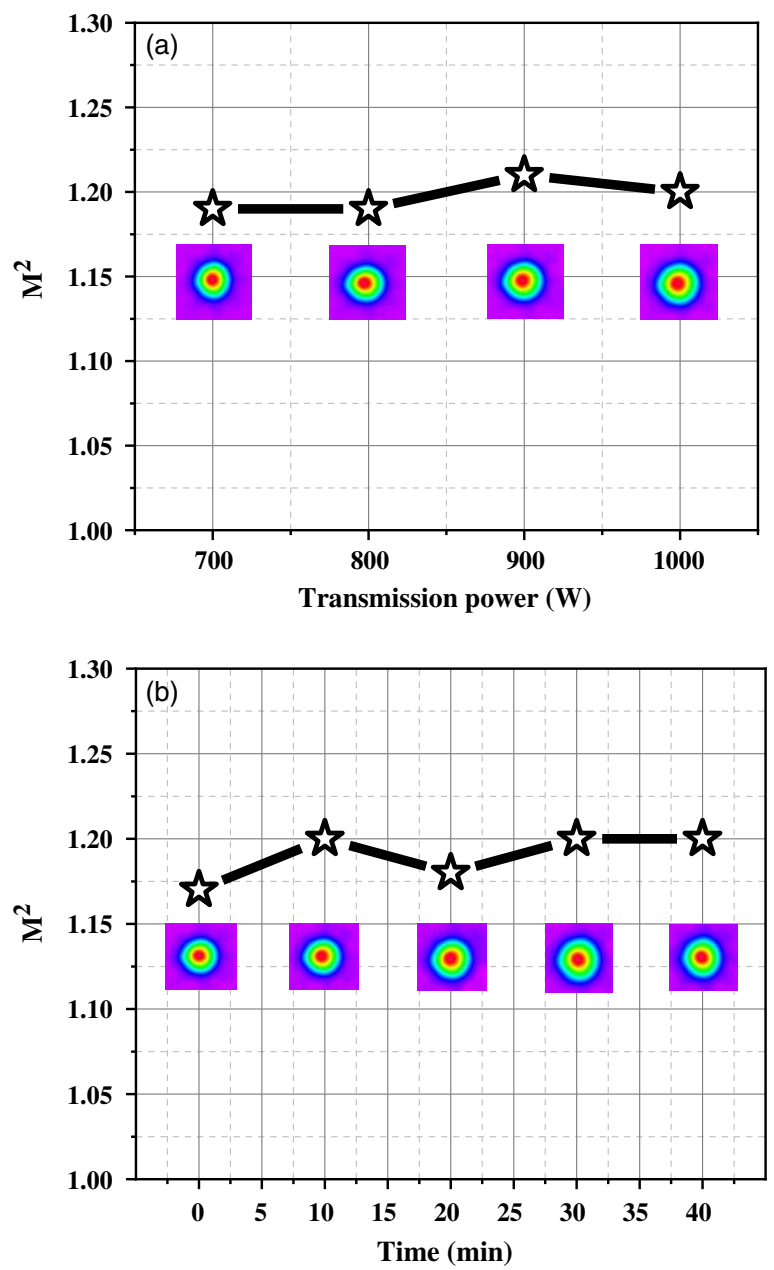

Fig. 5. (a) Beam quality $M^{2}$ versus transmission power; (b) beam quality $M^{2}$ versus time when the transmission power is $900 \mathrm{~W}$. Inset: beam profiles of waist. 
has a higher temperature of around $45.3^{\circ} \mathrm{C}$, the rear fiber has a lower temperature under the action of thermal expansion and cold contraction, and the input end of the HCF may have a minor change causing the overlap between the high power and the capillaries. Therefore, the output power decreased. But, because of the fixture of the end-cap, the change occurs slowly. As the transmission power dips to $915 \mathrm{~W}$, the power changes periodically with the power of the laser source, and the power fluctuation is less than $\pm 0.5 \%$. Compared with the condition above, the system can work stably when the transmission power is $915 \mathrm{~W}$. As the power decreased, the stability is also increased, which indicates that this method is an efficient coupling method for long working time at the $900 \mathrm{~W}$ power level.

Beam quality factor $M^{2}$ is measured using a laser quality monitor from PRIMES. At the output end of the HCF, a planoconvex lens with the focus of $50 \mathrm{~mm}$ is used to collimate the beam, and the results are shown in Fig. 5. Figure 5(a) shows the $M^{2}$ at different transmission power from 700 to $1000 \mathrm{~W}$, and, as the transmission power increased, the $M^{2}$ stabilized at around 1.2. Compared with the 1.17 of the laser source, $M^{2}$ keeps well after the light transmits in the HCF. As the system can keep working stably at the $900 \mathrm{~W}$ power level, $M^{2}$ versus time is measured when the transmission power is $900 \mathrm{~W}$, and the result is shown in Fig. 5(b). During the testing time, $M^{2}$ is relatively stable at around 1.2, which indicates that $M^{2}$ after transmitting in the HCF can remain stable at a high-power level.

\section{Conclusions}

In conclusion, through splicing the HCF with the end-cap, a high-efficiency and stable coupling method for HCF is achieved. The maximum transmission power reaches $1021 \mathrm{~W}$, as the input power is $1167 \mathrm{~W}$ with a high efficiency of $87.5 \%$. Besides, the system can keep good power stability and beam quality at the power level of $900 \mathrm{~W}$. The HCF does not have obvious heat positions apart from the exposed cladding applied with matching paste. By further optimizing the system, improving the coupling efficiency, and improving the heat treatment technology, the HCF has the potential to transmit higher power, which is significant for the development of high-power FGLs.

\section{Acknowledgement}

The authors thank Jonathan Knight from Bath University for supporting the HCF. This work was supported by the Outstanding Youth Science Fund of Hunan Provincial Natural Science Foundation (No. 2019JJ20023), National Natural Science Foundation of China (NSFC) (Nos. 11974427 and 12004431), State Key Laboratory of Pulsed Power Laser (Nos. SKL2020ZR05 and SKL2021ZR01), Science and Technology Innovation Program of Hunan Province (No. 2021RC4027), and Postgraduate Scientific Research Innovation Project of Hunan Province (No. CX20200017).

\section{References}

1. P. Russell, P. Hölzer, W. Chang, A. Abdolvand, and J. C. Travers, "Hollowcore photonic crystal fibres for gas-based nonlinear optics," Nat. Photonics 8 , 278 (2014)

2. A. V. V. Nampoothiri, A. M. Jones, C. Fourcade-Dutin, C. Mao, N. Dadashzadeh, B. Baumgart, Y. Wang, M. Alharbi, T. Bradley, and N. Campbell, "Hollow-core optical fiber gas lasers (HOFGLAS): a review [Invited]," Opt. Mater. Express 2, 948 (2012).

3. H. Li, W. Huang, Y. Cui, Z. Zhou, and Z. Wang, " $3 \mathrm{~W}$ tunable $1.65 \mathrm{~m}$ fiber gas Raman laser in $\mathrm{D}_{2}$-filled hollow-core photonic crystal fibers," Opt. Laser Technol. 132, 106474 (2020).

4. M. S. Astapovich, A. V. Gladyshev, M. M. Khudyakov, A. F. Kosolapov, M. E. Likhachev, and I. A. Bufetov, "Watt-level nanosecond $4.42-\mu \mathrm{m}$ Raman laser based on silica fiber," IEEE Photonics Technol. Lett. 31, 78 (2019).

5. L. Cao, S. Gao, Z. Peng, X. Wang, and P. Wang, "High peak power $2.8 \mu \mathrm{m}$ Raman laser in a methane-filled negative-curvature fiber," Opt. Express 26, 5609 (2018).

6. A. V. Gladyshev, A. F. Kosolapov, M. M. Khudyakov, Y. Yatsenko, A. N. Kolyadin, A. A. Krylov, A. Pryamikov, A. S. Biriukov, M. E. Likhachev, and I. A. Bufetov, "2.9, 3.3 and $3.5 \mu \mathrm{m}$ Raman lasers based on revolver hollow-core silica fiber filled by $\mathrm{H}_{2} / \mathrm{D}_{2}$ gas mixture," IEEE J. Sel. Top. Quantum Electron. 24, 0903008 (2018).

7. F. C. Ouny, B. J. Mangan, A. V. Sokolov, and F. Benabid, "High power 55 watts CW Raman fiber-gas-laser," in Conference on Lasers and ElectroOptics (CLEO) (2010), paper CTuM3.

8. F. Benabid, "Stimulated Raman scattering in hydrogen-filled hollow-core photonic crystal fiber," Science 298, 399 (2002).

9. F. B. A. Aghbolagh, V. Nampoothiri, B. Debord, F. Gerome, L. Vincetti, F. Benabid, and W. Rudolph, "Mid IR hollow core fiber gas laser emitting at 4.6 um," Opt. Lett. 44, 383 (2019).

10. Y. Cui, W. Huang, Z. Wang, M. Wang, Z. Zhou, Z. Li, S. Gao, Y. Wang, and P. Wang, "4.3 $\mu \mathrm{m}$ fiber laser in $\mathrm{CO}_{2}$ fibers," Optica 6, 951 (2019).

11. Z. Zhou, N. Tang, Z. Li, W. Huang, Z. Wang, W. Wu, and W. Hua, "Highpower tunable mid-infrared fiber gas laser source by acetylene-filled hollowcore fibers," Opt. Express 26, 19144 (2018).

12. $\mathrm{M} . \mathrm{Xu}, \mathrm{Y}$. Fei, and $\mathrm{K}$. Jonathan, "Mid-infrared $1 \mathrm{~W}$ hollow-core fiber gas laser source," Opt. Lett. 42, 4055 (2017).

13. M. R. A. Hassan, F. Yu, W. J. Wadsworth, and J. C. Knight, "Cavity-based mid-IR fiber gas laser pumped by a diode laser," Optica 3, 218 (2016).

14. A. M. Jones, A. V. V. Nampoothiri, A. Ratanavis, T. Fiedler, and W. Rudolph, "Mid-infrared gas filled photonic crystal fiber laser based on population inversion," Opt. Express 19, 2309 (2011).

15. X. Zhu, D. Wu, Y. Wang, F. Yu, Q. Li, Y. Qi, J. Knight, S. Chen, and L. Hu, "Delivery of CW laser power up to 300 watts at $1080 \mathrm{~nm}$ by an uncooled lowloss anti-resonant hollow-core fiber," Opt. Express 29, 1492 (2021).

16. S. H. Drich, J. Rothhardt, S. Demmler, M. Tschernajew, A. Hoffmann, M. Krebs, A. Liem, O. D. Vries, M. Pl Tner, and S. Fabian, "Scalability of components for kW-level average power few-cycle lasers," Appl. Opt. 55, 1636 (2016).

17. F. Benabid, F. Couny, J. Knight, T. Birks, and P. Russell, "Compact, stable and efficient all-fibre gas cells using hollow-core photonic crystal fibres," Nature 434, 488 (2005).

18. P. S. Light, F. Couny, and F. Benabid, "Low optical insertion-loss and vacuum-pressure all-fiber acetylene cell based on hollow-core photonic crystal fiber," Opt. Lett. 31, 2538 (2006).

19. K. Z. Aghaie, M. J. F. Digonnet, and S. Fan, "Optimization of the splice loss between photonic-bandgap fibers and conventional single-mode fibers," Opt. Lett. 35, 1938 (2010).

20. S. Gao, Y. Wang, C. Tian, and P. Wang, "Splice loss optimization of a photonic bandgap fiber via a high V-number fiber," IEEE Photonics Technol. Lett. 26, 2134 (2014).

21. H. Li, W. Huang, W. Pei, Z. Zhou, Y. Cui, M. Wang, and Z. Wang, "All-fiber gas Raman laser oscillator," Opt. Lett. 46, 5208 (2021).

22. W. Pei, H. Li, W. Huang, M. Wang, and Z. Wang, "Hydrogen molecules rotational stimulated Raman scattering in all-fiber cavity based on hollow-core photonic crystal fibers," Crystals 11, 711 (2021). 
23. W. Pei, H. Li, W. Huang, M. Wang, and Z. Wang, “All-fiber tunable pulsed $1.7 \mu \mathrm{m}$ fiber lasers based on stimulated Raman scattering of hydrogen molecules in hollow-core fibers," Molecules 26, 4561 (2021).

24. W. Pei, H. Li, W. Huang, M. Wang, and Z. Wang, "Pulsed fiber laser oscillator at $1.7 \mu \mathrm{m}$ by stimulated Raman scattering in $\mathrm{H}_{2}$-filled hollow-core photonic crystal fibers," Opt. Express 29, 33915 (2021).

25. W. Pei, H. Li, W. Huang, M. Wang, and Z. Wang, "All-fiber gas Raman laser by $\mathrm{D}_{2}$-filled hollow-core photonic crystal fibers," Photonics 8, 382 (2021).

26. X. Zheng, B. Debord, L. Vincetti, B. Beaudou, and F. A. Benabid, "Fusion splice between tapered inhibited coupling hypocycloid-core Kagome fiber and SMF," Opt. Express 24, 14642 (2016).

27. R. Zeltner, S. Xie, R. Pennetta, and P. Russell, "Broadband, lensless and optomechanically stabilised coupling into microfluidic hollow-core photonic crystal fiber using glass nanospike," ACS Photonics 4, 378 (2016).

28. W. Huang, Y. Cui, Z. Zhou, Z. Li, Y. Chen, and Z. Wang, “Towards all-fiber structure pulsed mid-infrared laser by gas-filled hollow-core fibers," Chin. Opt. Lett. 17, 091402 (2019).
29. Y. Cui, Z. Zhou, W. Huang, Z. Li, and Z. Wang, "Quasi-all-fiber structure CW mid-infrared laser emission from gas-filled hollow-core silica fibers," Opt. Laser Technol. 121, 105794 (2019).

30. W. Huang, Y. Cui, X. Li, Z. Zhou, and Z. Wang, "Low-loss coupling from single-mode solid-core fibers to anti-resonant hollow-core fibers by fiber tapering technique," Opt. Express 27, 37111 (2019).

31. R. Yu, C. Wang, F. Benabid, K. S. Chiang, and L. Xiao, "Robust mode matching between structurally dissimilar optical fiber waveguides," ACS Photonics 8, 857 (2021).

32. C. Wang, R. Yu, B. Debord, F. Gérôme, F. Benabid, K. S. Chiang, and L. Xiao, "Ultralow-loss fusion splicing between negative curvature hollow-core fibers and conventional SMFs with a reverse-tapering method," Opt. Express 29, 22470 (2021).

33. J. Shi, X. Ye, Y. Cui, W. Huang, H. Li, Z. Zhou, M. Wang, Z. Chen, and Z. Wang, "All-fiber gas cavity based on anti-resonant hollow-core fibers fabricated by splicing with end caps," Photonics 8, 371 (2021). 